\title{
Cardiac inflammatory myofibroblastic tumor as a rare cause of aortic regurgitation: A case report
}

\author{
Jang Wen Su, MBBS, MRCS, MMed, ${ }^{a}$ Michael George Caleb, MBBS, FRCS, FAMS ${ }^{\mathrm{c}}$ Ru San Tan, MBBS, FRCP, FAMS, \\ Adrian Fatt Hoe Low, MBBS, FRCP, FAMS, ${ }^{d}$ and Chong Hee Lim, MBBS, FRCS, FAMS, ${ }^{\text {a }}$ Singapore
}

I nflammatory myofibroblastic tumor (IMT), also known as plasma cell granuloma, is a rare lesion reported in young patients. ${ }^{1}$ IMT poses a challenge in diagnosis even for the most experienced pathologist, cardiologist, and cardiac surgeon. $^{2}$ Its precise biologic nature and clinical characteristics are still uncertain. It was first reported to involve the lung but is now known to occur in almost every major organ of the body. ${ }^{3} \mathrm{We}$ report a patient with a cardiac IMT diagnosis, involving the aortic root and right ventricular outflow tract, causing mechanical distortion of the aortic valve with resultant severe aortic regurgitation. We believe this is the first reported case of IMT as a direct cause of aortic regurgitation.

\section{Case Report}

A 48-year-old woman initially presented with fever lasting 3 months. During the workup, end-diastolic murmur in the right parasternal region was found. She was otherwise asymptomatic. Her heart rate was in sinus rhythm at 88 beats/min, and her blood pressure was $110 / 40 \mathrm{~mm} \mathrm{Hg}$. No lymphadenopathy or hepatosplenomegaly was detected.

Serial full blood count showed normal white blood cell count with normal neutrophil and lymphocyte differentials. Liver and renal function test results were unrevealing. The main abnormal biochemical markers were increased erythrocyte sedimentation rate $(130 \mathrm{~mm} / \mathrm{h})$ and $\mathrm{C}$-reactive protein $(43 \mathrm{mg} / \mathrm{L})$. Myeloma screen was negative, serial blood cultures showed no growth of bacteria, and acid-fast bacilli was negative.

Transthoracic echocardiogram confirmed severe aortic regurgitation with a large transmural cardiac tumor at the root of the aorta and the right ventricular outflow tract, extending to encircle the proximal pulmonary artery. The mass was believed to have impinged on the aortic valve, causing mechanical aortic regurgitation. A moderate amount of pericardial effusion was also reported. Cardiac computed tomography and magnetic resonance imaging

From the Departments of Cardiothoracic Surgery a and Cardiovascular Medicine, ${ }^{\mathrm{b}}$ National Heart Center, Singapore General Hospital; and Departments of Cardiothoracic and Vascular Surgery ${ }^{\mathrm{c}}$ and Cardiology, ${ }^{\mathrm{d}}$ The Heart Institute, National University Hospital, Singapore.

Received for publication Jan 5, 2006; accepted for publication March 2, 2006.

Address for reprints: Address for reprints: Jang Wen Su, MBBS, MRCS, MMed, Registrar, Department of Cardiothoracic Surgery, National Heart Centre, Mistri Wing, 17 Third Hospital Avenue, Singapore 168752 (Email: bottle1001@yahoo.com).

J Thorac Cardiovasc Surg 2006;132:150-1

$0022-5223 / \$ 32.00$

Copyright $@ 2006$ by The American Association for Thoracic Surgery doi:10.1016/j.jtcvs.2006.03.011 were also performed, which confirmed the echocardiogram findings (Figure 1).

In view of the fever, increased inflammatory markers, and aortic regurgitation with cardiac mass at the aortic root, treatment with intravenous penicillin and gentamicin was commenced for presumptive diagnosis of infective endocarditis.

Subsequently, the patient underwent upper sternotomy and biopsy of the mass. Intraoperatively, we found moderate amount of turbid pericardial effusion. There was a firm mass at the aortopulmonary space involving the root of the aorta and the right ventricular outflow tract. The surface of the mass and adjacent pericardium appeared inflamed. Frozen section of the biopsy tissue showed dense plasma cell infiltration.

Bone marrow trephine biopsy, in view of plasma cell infiltrates, was suggestive of an inflammatory process.

On the tenth postoperative day, the patient experienced sustained ventricular tachycardia requiring direct-current cardioversion. Otherwise, she made an uneventful recovery.

The tissue cultures for bacteria, acid-fast bacilli, and fungus were all negative.

Repeat transthoracic echocardiogram showed no regression of the cardiac mass and no resolution of aortic regurgitation. She continued taking antibiotics for 4 weeks. Throughout the course of

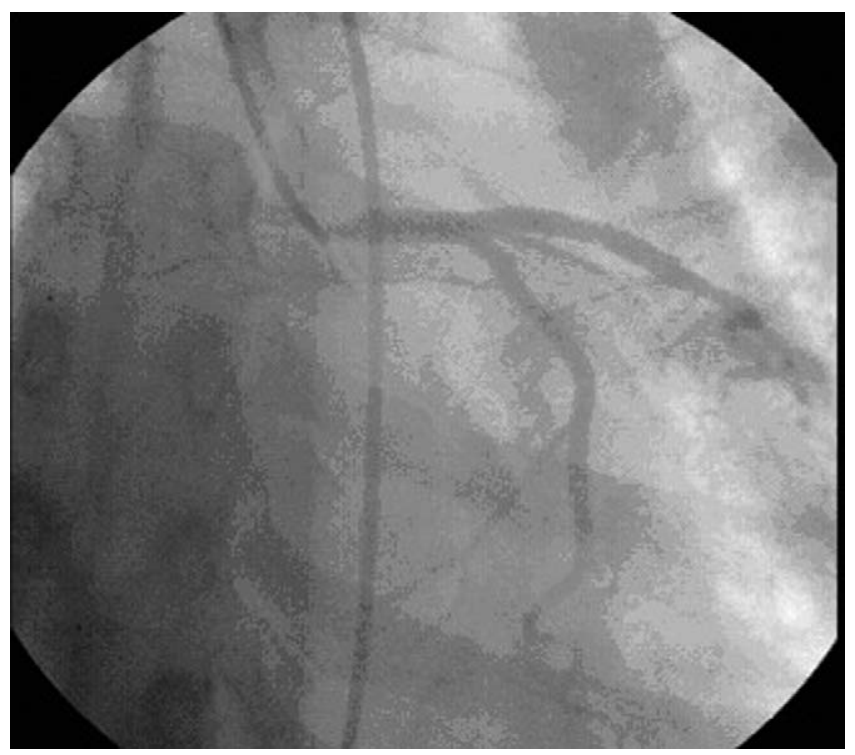

Figure 1. Infiltrative mediastinal mass involving mainly right ventricular outflow tract and lateral and posterior walls of left ventricular outflow tract. The pulmonary artery wall was also infiltrated. 


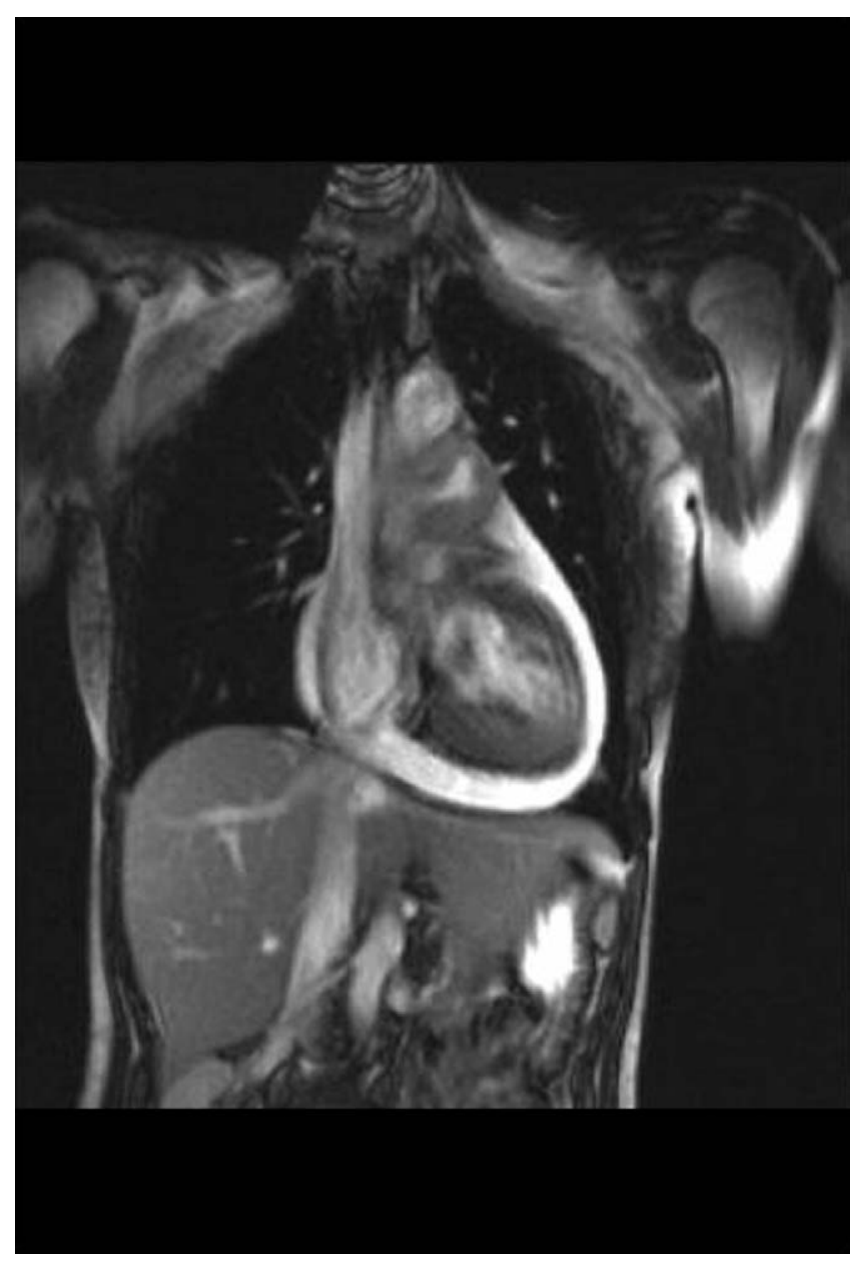

Figure 2. Cardiac catheterization showing $\mathbf{9 0} \%$ ostial left main stenosis.

treatment, the patient's white blood cell count never increased; however, her erythrocyte sedimentation rate and C-reactive protein were persistently elevated.
Antineutrophil cytoplasmic antibodies, antinuclear antibodies, rheumatoid factor, and tumor markers were insignificant. A clinical diagnosis of IMT was made, and treatment with prednisolone was begun.

Two months later, the patient had an anterior myocardial infarction. Cardiac catheterization revealed pinching of the ostium of the left main coronary artery (Figure 2). This was secondary to constriction from the exuberant fibrosis around the aortic root. The aortic regurgitation was severe. In view of the patient's complicated medical history she underwent percutaneous angioplasty with stenting using a Liberte bare metal stent (Boston Scientific Corp, Natick, Mass). She subsequently improved, and anti-failure therapy was continued. Ten days later she underwent a repeat open biopsy that revealed only tissue fibrosis with no evidence of malignancy. Steroid therapy was resumed postoperatively. We do not believe that the aortic regurgitation will further improve because the aortic root that was previously distorted by tumor invasion is now affected by fibrosis. The mechanical element of aortic regurgitation remains. However, after steroid therapy, the integrity of the aorta has improved and become firm enough to allow a planned aortic valve replacement. This is in comparison with the extremely friable aortic root at the first surgical encounter.

IMT is a rare cardiac tumor. However, in the inflammatory syndrome, it must be considered in the differential diagnosis. Our patient presented with fever of unknown origin, which subsequently led to the discovery of the aortic regurgitation and cardiac tumor. Throughout the whole course of management, IMT highlights the difficulty in attaining the diagnosis. Thorough and extensive investigations are paramount in identifying this rare condition so appropriate treatment can be instituted.

\section{References}

1. de Montpreville VT, Serraf A, Aznag H, Nashashibi N, Planche C, Dulmet E. Fibroma and inflammatory myofibroblastic tumour of the heart. Ann Diagn Pathol. 2001;5:335-42.

2. Kasis A, Chukwuemeka AO, Vecht JA, Ibrahim MF, Young CP. An unusual cause of ventricular tachycardia. Int J Clin Pract. 2004;58: 807-8.

3. Liz L, Cerilli LA, Wick MR. Inflammatory pseudotumour (myofibroblastic tumour) of the heart. Ann Diagn Pathol. 2002;6:116-21. 